\title{
Diferentes critérios de definição da periodontite em puérperas jovens e sua associação com o baixo peso ao nascer
}

\author{
Different criteria for periodontitis definition in young mothers and its association with \\ low birth weight
}

\author{
Mércia Sacramento dos Santos ${ }^{1}$, Laíra Sá Lopes², Isaac Gomes-Filho³, Johelle Passos-Soares ${ }^{4}$, Maria \\ Cristina Teixeira Cangussu ${ }^{5 *}$, Maria Isabel Pereira Vianna ${ }^{6}$
}

\begin{abstract}
${ }^{1}$ Doutoranda em Odontologia e Saúde, Faculdade de Odontologia, Universidade Federal da Bahia (UFBA); ${ }^{2}$ Mestre em Saúde Comunitária, Instituto de Saúde Coletiva, Universidade Federal da Bahia (UFBA); ${ }^{3}$ Doutor em Periodontia, Universidade de São Paulo (USP), Professor Titular da Universidade Estadual de Feira de Santana (UEFS); ${ }^{4}$ Doutora em Saúde Comunitária, Instituto de Saúde Coletiva, Universidade Federal da Bahia (UFBA), Professora Adjunto,

Faculdade de Odontologia da Universidade Federal da Bahia (UFBA); ${ }^{5}$ Doutora em Saúde Comunitária pelo Instituto de Saúde Coletiva, Universidade Federal da Bahia (UFBA), Professora Titular da Faculdade de Odontologia, Universidade Federal da Bahia (UFBA); ${ }^{6}$ Doutora em Saúde Pública pela Universidade de São Paulo (USP), Professora Titular da Faculdade de Odontologia, Universidade Federal da Bahia (UFBA)
\end{abstract}

\begin{abstract}
Resumo
Objetivo: comparar três critérios de definição da periodontite na associação da doença periodontal com o baixo peso ao nascer (BPN) em uma população de puérperas jovens de Salvador, BA. Metodologia: aplicaram-se três diferentes critérios de definição da periodontite a uma amostra de 202 mães de bebês com peso $\geq 2.500 \mathrm{~g}$ (controles) e de 97 mães de bebês com BPN (casos) pertencentes a uma base de dados de um estudo caso-controle prévio. Para a análise da associação entre periodontite e BPN, construíram-se modelos para os três critérios. A regressão logística não-condicional foi realizada para estimar a Odds Ratio (OR) baseada em Intervalos de Confiança a 95\%. Resultados: a idade média das mães foi de 26,29 anos. A maioria declarou-se negra/ parda $(92.64 \%)$, não fumante $(94,65 \%)$ e possuir renda de até um salário-mínimo $(61,20 \%)$. A ocorrência da periodontite variou de $22,41 \%$ a $94,31 \%$, segundo o critério usado. Estimando-se a OR ajustada, a periodontite definida pelo critério 1 [ORajust. = 0,94, IC $(95 \%)=0,56-1,56]$, critério 2 [ORajust $=1,18, I C(95 \%)=0,65-2,13$ ] e critério 3 [ORajust= $0,87, I C(95 \%)=0,37-3,22]$ não esteve associada com o BPN. Conclusão: flexibilizar o critério de definição interferiu na magnitude das medidas de ocorrência, mas não influenciou a análise de associação entre a periodontite e o BPN.
\end{abstract}

Palavras-chave: Periodontite. Diagnóstico periodontal. Baixo peso ao nascer. Adultos jovens.

\begin{abstract}
Objective: to compare three criteria for periodontitis definition and its association with Low Birth Weight (LBW) in a population of young mothers, in the city of Salvador - BA. Methods: Three different definitions for periodontitis were applied to sample of 202 mothers of newborns (NB) weighing $\geq 2.500 \mathrm{~g}$ (controls) and 97 mothers of NB with LBW (cases) belonging to database from a previous case-control study. For the analysis of the association between periodontitis and LBW, were created models for three criteria. An unconditional logistic regression was used to estimate the odds ratio (OR) with 95\% confidence interval. Results: the average age of mothers was 26,29 years. Most respondents identified themselves as black or brown (92.64\%), 94,65\% nonsmokers (94,65\%) and have income up to 1 minimum wage (61.20\%). The occurrence of periodontitis varied between $22,41 \%$ to $94,31 \%$ according to the criteria. When estimating adjusted $O R$, the periodontitis defined by criterion 1 [ORajus $=0,94, I C(95 \%)=0,56-1,56]$, criterion 2 [ORajus $=1,18, I C(95 \%)=0,65-2,13]$ and criterion 3 [ORajus $=0,87, I C(95 \%)=0,37-3,22]$ did not demonstrate association with LBW. Conclusion: to flex periodontitis' criteria modified the magnitude of measures of occurrence but did not influence the association analysis between periodontitis and the BPN.

Keywords: Periodontitis. Periodontal diagnosis. Low birth weight. Young adults
\end{abstract}

\section{INTRODUÇÃO}

As doenças periodontais englobam uma série de alterações patológicas resultantes da infecção do periodonto por bactérias patógenas do biofilme dental. Em determinados indivíduos e sítios dentários, a infecção pode progredir para a perda óssea, sendo então denomi-

Correspondente/Corresponding: *Maria Cristina Teixeira Cangussu End: Avenida Araújo Pinho, 62 , 60 andar, Canela, Salvador-BA, CEP: 41110-150. Tel: (71) 32838990 -E-mail: cangussu@ufba.b nada de periodontite (LOURO et al., 2001). A periodontite apresenta variabilidade clínica (GOMES-FILHO et al., 2006), causalidade, patogênese e desenvolvimento complexos devido à influência da resposta imune individual (CORTELLI JUNIOR; CORTELLI, 2003) frente às condições ambientais.

Complexa também é a definição da doença, devido a ampla variedade de métodos diagnósticos e critérios de definição, que distintos para fins clínicos e científicos, 
resultam muitas vezes, na obtenção de dados divergentes e na incapacidade de comparação entre os resultados das pesquisas.

Desde os anos 90, evidências indicam o potencial de contribuição das bactérias periodontopatógenas, seus produtos e/ou mediadores inflamatórios desencadeados em eventos sistêmicos, dentre os quais as complicações gestacionais, prematuridade e o baixo peso ao nascer (OFFENBACHER et al., 1996). No entanto, a literatura ainda não foi capaz de estabelecer um critério diagnóstico padrão para o estudo da periodontite enquanto exposição, por desconhecer os sinais clínicos que meIhor representariam o seu potencial de dano sistêmico, além da magnitude necessária para que o dano ocorra (SCHENKEIN et al., 2012).

Gomes-Filho et al. (2006) sugerem que definições da periodontite a serem utilizadas em análises de associação com condições sistêmicas devem englobar a mensuração do nível de inserção clínica (NIC), profundidade de sondagem (PS) e a presença de sangramento à sondagem (SS) visando um critério mais restritivo e específico. No entanto, sabe-se que uma alta especificidade culmina na perda de sensibilidade e possível exclusão de casos limítrofes, situação que reduziria a ocorrência da doença atribuída a uma população, mas poderia não representar seu verdadeiro quadro (LOURO et al., 2001).

Considerando que o NIC, medida clínica mais comum nos estudos periodontais, revela apenas a história cumulativa da doença e que a PS e SS isoladamente, não podem determinar a presença de atividade da doença (OFFENBACHER et al., 1996), entende-se que os critérios disponíveis na literatura parecem apresentar aplicabilidade para idades específicas (WHO, 2011).

Nascidos vivos com baixo peso ao nascer (BPN), ou seja, peso inferior a $2.500 \mathrm{~g}$ são considerados um problema de saúde pública mundial (WHO, 2011). Posto que sua ocorrência é um importante preditor de morte neonatal e sequelas permanentes (LOURO et al., 2001; WHO, 2011), cujos tratamentos são onerosos para o Estado (LOURO et al., 2001; OFFENBACHER et al., 1996). O baixo peso ao nascer está associado a hábitos de consumo, fatores sociais e saúde (ORTELAN; NERI; BENICIO, 2020). Também está associado à ocorrência de infecções intrauterinas e à atuação de mediadores inflamatórios locais e sistêmicos ascendestes de infecções da vagina, do colo do útero ou pela disseminação hematogênica, oriundos de infecções alheias ao trato urogenital (SANZ; KORNMAN, 2013). A periodontite potencialmente participaria nestes processos através da indução inflamatória em sua progressão e da participação de citocinas inflamatórias comuns a ambos os eventos (POZO et al., 2016).

A padronização dos critérios diagnósticos da periodontite como exposição propiciaria adequada comparação de pesquisas futuras para elucidar a relação existente entre a periodontite e o BPN (MANNEM; CHAVA, 2011), aumentado suas estratégias de enfrentamento.
Diante desta lacuna e das evidências de que a força da associação da periodontite com complicações gestacionais parece estar associada tanto as características da população analisada (IDE; PAPAPANOU, 2013), aos limiares de mensuração da periodontite, quanto aos critérios escolhidos para defini-la (GOMES-FILHO et al., 2009; IDE; PAPAPANOU, 2013), objetivou-se avaliar o comportamento de diferentes critérios de diagnóstico da periodontite na análise da sua associação com o baixo peso ao nascer em uma população de mulheres adultas jovens.

\section{METODOLOGIA}

\section{População Do Estudo}

A amostra foi composta por 299 indivíduos - 97 casos e 202 controles, e calculada com base em parâmetros de distribuição da periodontite entre puérperas disponíveis na literatura e a partir de uma base de dados pré-existente oriunda de um estudo observacional, retrospectivo e do tipo caso-controle intitulado "Interação biológica entre doença periodontal e hiperglicemia não diabética materna para a ocorrência do baixo peso ao nascer", aprovado pelos comitês de ética em pesquisa da Faculdade de Odontologia (CAAE - 0024.0.368.368-09) e da Maternidade Climério de Oliveira, ambas unidades pertencentes à Universidade Federal da Bahia.

A coleta de dados do estudo original ocorreu no período de abril a dezembro de 2011 em uma maternidade pública de referência no atendimento à gravidez de risco e patologias de recém-nascidos e em uma maternidade/ escola de universidade pública. Ambas pertencentes ao Sistema Único de Saúde e ao município de Salvador-Ba.

O Grupo Caso foi constituído de mães de crianças nascidas com peso inferior a $2.500 \mathrm{~g}$, nas duas unidades hospitalares. A composição do Grupo Controle foi de mães de recém-nascidos dos mesmos hospitais onde foram escolhidos os casos, com peso igual ou superior a $2.500 \mathrm{~g}$.

\section{Procedimentos de Coleta Dos Dados}

O banco foi composto a partir de dados dos prontuários médicos dos hospitais, das entrevistas com as mães e exame clínico periodontal, após aceitação e assinatura do termo de consentimento livre e esclarecido. O questionário utilizado abrangeu informações sobre identificação do recém-nascido e da mãe, história médica gestacional, uso de medicamentos na gestação atual, dados do pré-natal e do parto; dados antropométricos da mãe, higiene bucal e atenção odontológica na gestação atual; percepção sobre saúde bucal, hábitos de vida na gestação atual, qualidade do pré-natal, dados do recém-nascido e do nascimento. O peso dos recém-nascidos foi obtido do livro de registro de nascimento das maternidades.

As puérperas foram também submetidas a exame clínico periodontal completo (com o auxílio de espelho 
bucal e sonda periodontal manual do tipo Williams) realizado, de modo cego, por um único periodontista devidamente treinado.

Realizou-se a mensuração da profundidade de sondagem em seis diferentes locais para cada dente (nos ângulos mésio-vestibular, mésio-lingual, disto-vestibular e distolingual, na região médio-vestibular e uma na região médio-lingual). Obteve-se a medida de inserção clínica através da soma dos valores da PS de sulco/bolsa e medidas de recessão ou hiperplasia gengivais (CRUZ et al., 2005). Já o índice de sangramento a sondagem foi determinado observando a presença do sangramento em 10 segundos após a remoção da sonda milímetrada do sulco ou bolsa no registro da PS (CRUZ et al., 2005).

Não foram incluídas no estudo as mães com diabetes mellitus, doenças cardiovasculares, as que necessitavam de profilaxia antibiótica para os procedimentos odontológicos e as que relatassem tratamento periodontal durante a gestação.

\section{Definição de Critérios de Periodontite}

A escolha dos critérios aqui adotados norteou-se pelo fato de dois deles serem amplamente empregados em estudos dessa linha na população brasileira (CRUZ et al., 2005; GOMES-FILHO et al., 2006; GOMES-FILHO et al., 2007; SUSIN et al., 2011) e o outro ser recomendado pela Academia Americana de Periodontia em parceria com o Centro de Controle de Doenças e Prevenção para situações de levantamento populacional da doença (EKE et al., 2012). As descrições de cada critério encontram-se no quadro 1.

Quadro 1- Critérios de definição da periodontite adotados no estudo.

\begin{tabular}{|l|l|l|}
\hline Critério & Autores & Definição \\
\hline 01 & Eke et al. 2012 & $\begin{array}{l}\text { Ao menos dois sítios interproximais } \\
\text { de dentes diferentes com perda de } \\
\text { inserção clínica maior ou igual a 4 } \\
\text { mm ou dois sítios interproximais em } \\
\text { dentes diferentes com profundidade } \\
\text { de sondagem maior ou igual a 5 mm. }\end{array}$ \\
\hline 02 & $\begin{array}{l}\text { Gomes-Filho et al. } \\
2006\end{array}$ & $\begin{array}{l}\text { Quatro ou mais dentes com um ou mais } \\
\text { sítios com profundidade de sondagem } \\
\text { maior ou igual a 4 mm, com perda de } \\
\text { inserção clínica maior ou igual a 3 mm no } \\
\text { mesmo sítio e presença de sangramento } \\
\text { a sondagem. }\end{array}$ \\
\hline 03 & Susin et al. 2011 & $\begin{array}{l}\text { Dois ou mais dentes apresentando sítios } \\
\text { interproximais com perda de inserção } \\
\text { clínica maior ou igual a 3 mm. }\end{array}$ \\
\hline
\end{tabular}

Fonte: Autoria própria

\section{Procedimentos de Análise dos Dados}

A partir das respostas registradas nos questionários $\mathrm{e}$ dos dados do periodontograma foram obtidas a variável dependente, o BPN (grupo caso e grupo controle), e a variável independente principal, indicada como presença de periodontite. A partir de um modelo preditivo definido no estudo mãe para a associação entre a periodontite e o BPN, foram consideradas as seguintes co-variáveis: a faixa de idade ( 26 a 45 anos e 14 a 25 anos), o nível de escolaridade materna (Analfabeta ou até o 8 ano fundamental e do 9o ano fundamental ou mais), o hábito de fumar e o número de consultas pré-natais realizadas. Os pontos de corte adotados foram traçados a partir das medianas dos dados e baseados em estudos anteriores.

Procedeu-se a análise descritiva das variáveis sociodemográficas (frequências simples, medidas de tendência central e dispersão e os testes Qui-quadrado de Pearson e TStudent com nível de significância de 5\%). Odds Ratio e respectivos intervalos de confiança a 95\% (IC95\%) foram obtidos para a ocorrência de BPN e periodontite considerando-se cada um dos três critérios utilizados. Um modelo preditivo foi definido e aplicado para cada um dos três critérios, então avaliados comparativamente quanto a sua associação com o BPN, com base na literatura e nos resultados da análise estratificada. Realizou-se a análise multivariada com regressão logística não condicional. Para a inferência estatística utilizou-se o IC ao nível de 95\%. A presença de modificadoras de efeito foi verificada pelo teste de Razão de Máxima Verossimilhança $(p<0,05)$, e o papel de variáveis selecionadas de confundimento foi avaliado empregando-se a estratégia backward, sendo estas consideradas a partir de bases teóricas e empíricas.

Estes procedimentos foram conduzidos no programa estatístico STATA/SE (Stata Corp. College Station, Estados Unidos), versão 10.0.

$O$ estudo original foi financiado pela Fundação de Amparo à Pesquisa do Estado da Bahia - FAPESB sob processo EFP_00001327.

\section{RESULTADOS}

A amostra constou de 299 puérperas, com idade média de 26,29 anos, desvio de 6,60 (IC 95\%= 25,34$27,04)$. O peso ao nascer dos recém-nascidos variou de $660 \mathrm{~g}$ a $4.610 \mathrm{~g}$, apresentando uma média de $2.849 \mathrm{~g}(\mathrm{dp}=$ $823,9 \mathrm{~g}$ ). As gestações com período igual ou superior a 36 semanas representaram $80,94 \%(n=242)$ da amostra. Analisando as variáveis de saúde das puérperas, observou-se que $93,31 \%(n=279)$ destas realizaram acompanhamento pré-natal e que o número médio de consultas realizadas foi de $6,01(\mathrm{dp}=3,23)$.

A Tabela 1 apresenta alguns dados sociodemográficos e de saúde dos casos e controles. Pode se observar que a variável pré-natal categorizada indicou que $87,13 \%$ $(n=176)$ dos indivíduos do grupo controle e $64,95 \%$ $(n=63)$ dos casos realizaram quatro ou mais consultas, sendo essas diferenças estatisticamente significantes. 
As demais variáveis como hábito de fumar, a idade materna e o nível de escolaridade materna não estiveram associadas ao BPN.

A ocorrência de periodontite nas puérperas variou de acordo com o critério de definição da doença utilizado.
Mediante emprego do critério $1,41,47 \%(n=126)$ dos indivíduos mostraram-se com periodontite. 0 critério 2 identificou $22,41 \%(n=67)$ de doentes, enquanto o maior percentual de acometidos (94,31\%) foi observado quando da utilização do critério 3.

Tabela 1 - Características sociodemográficas e de saúde dos casos e controles (números, percentuais e respectivos valor de p). Salvador, Bahia. (n=299).

\begin{tabular}{|c|c|c|c|c|c|}
\hline \multirow[t]{2}{*}{ Características } & \multicolumn{2}{|c|}{$\operatorname{Casos}^{*}(\mathrm{n}=97)$} & \multicolumn{2}{|c|}{ Controles $^{* *}(n=202)$} & \multirow[t]{2}{*}{ pj } \\
\hline & $\mathbf{n}$ & $\%$ & $\mathbf{n}$ & $\%$ & \\
\hline \multicolumn{6}{|l|}{ Idade materna } \\
\hline 26 a 25 anos & 51 & 52.58 & 104 & 51.48 & \\
\hline 14 a 25 anos & 46 & 47.42 & 98 & 48.52 & 0.86 \\
\hline \multicolumn{6}{|l|}{ Raça } \\
\hline Branca & 7 & 7.22 & 15 & 7,43 & \\
\hline Negra/parda & 90 & 92.78 & 187 & 95.57 & 0.95 \\
\hline \multicolumn{6}{|l|}{ Nível de escolaridade materna } \\
\hline 9o ano do EFa ou mais & 72 & 25.77 & 153 & 75.74 & \\
\hline Alfabetização ao $8^{\circ}$ ano do $\mathrm{EFa}^{\alpha}$ & 25 & 74.23 & 49 & 24.26 & 0.78 \\
\hline \multicolumn{6}{|l|}{ Renda Familiar } \\
\hline Acima de 1 salário-mínimo & 35 & 32.99 & 81 & 40.10 & \\
\hline Até 1 salário-mínimo & 62 & 67.01 & 121 & 59.90 & 0.50 \\
\hline \multicolumn{6}{|l|}{ Hábito de fumar } \\
\hline Não fumante & 91 & 94.79 & 192 & 95.05 & \\
\hline Fumante & 6 & 5.21 & 10 & 4.95 & 0.65 \\
\hline \multicolumn{6}{|l|}{ Consultas pré-natais } \\
\hline 04 consultas ou mais & 63 & 64.95 & 176 & 87.13 & \\
\hline Até 03 consultas & 34 & 35.05 & 26 & 12.87 & 0.00 \\
\hline \multicolumn{6}{|l|}{ Idade gestacional } \\
\hline$\geq 36$ semanas & 42 & 43.30 & 200 & 99.01 & \\
\hline$<36$ semanas & 55 & 56.7 & 2 & 0.99 & 0.50 \\
\hline
\end{tabular}

$\alpha$ EFa - Ensino fundamental

*Mães de crianças com peso ao nascer inferior a $2500 \mathrm{~g}$.

**Mães de crianças com peso ao nascer igual ou superior a $2500 \mathrm{~g}{ }^{\dagger}$ Significância estatística, $\mathrm{p} \leq 0.05$.

Fonte: Autoria própria

A condição de saúde bucal das puérperas nos grupos, segundo os três critérios de definição da periodontite adotados, é apresentada na Tabela 2. A periodontite ainda que analisada a partir de três diferentes critérios de definição para a doença não se mostrou associada ao BPN na análise bivariada, resultado que se manteve na análise multivariada. 
Tabela 2-Ocorrência da periodontite de acordo com os critérios de classificação da periodontite, Odds Ratio (OR) bruto e Intervalo de confiança de 95\% (IC95\%). Salvador, Bahia. ( $n=299)$.

\begin{tabular}{|c|c|c|c|c|c|c|c|c|}
\hline \multirow[t]{2}{*}{$\begin{array}{c}\text { Variáveis } \\
\text { independentes }\end{array}$} & \multicolumn{2}{|c|}{$\begin{array}{c}\text { Controles } \\
(n=97)\end{array}$} & \multicolumn{2}{|c|}{$\begin{array}{l}\text { Casos } \\
(n=202)\end{array}$} & \multirow[t]{2}{*}{$\begin{array}{l}\text { OR } \\
\text { bruta }\end{array}$} & \multicolumn{2}{|c|}{ IC (95\%) } & \multirow[t]{2}{*}{$\begin{array}{l}\mathbf{p} \\
*\end{array}$} \\
\hline & $n$ & (\%) & $\mathrm{n}$ & (\%) & & & & \\
\hline \multicolumn{9}{|l|}{$\begin{array}{l}\text { Critério } 1 \\
\text { (Eke et al. 2012) }\end{array}$} \\
\hline Não & 117 & 57.92 & 58 & 59.79 & 0.92 & $(0,56$ & $1,51)$ & 0,76 \\
\hline Sim & 85 & 42.08 & 39 & 40.21 & & & & \\
\hline \multicolumn{9}{|l|}{$\begin{array}{l}\text { Critério } 2 \\
\text { (Gomes-Filho et al. 2006) }\end{array}$} \\
\hline Não & 159 & 78.71 & 73 & 75.26 & 1.21 & $(0,69$ & $2,15)$ & 0,50 \\
\hline Sim & 43 & 21.29 & 24 & 24.74 & & & & \\
\hline \multicolumn{9}{|l|}{$\begin{array}{l}\text { Critério } 3 \\
\text { (Susin et al. 2011) }\end{array}$} \\
\hline Não & 11 & 5.45 & 6 & 6.19 & 0.87 & $(0,31$ & $2,43)$ & 0,79 \\
\hline Sim & 191 & 94.55 & 91 & 93.81 & & & & \\
\hline
\end{tabular}

Fonte: Autoria própria

$\mathrm{Na}$ análise de confundimento, apenas a variável número de consultas pré-natais se apresentou como confundidora, ocorrendo apenas no modelo onde foi utilizado o critério 3. Apesar disto, o ajuste foi realizado, considerando a base teórica, nos três modelos finais. $\mathrm{Na}$ Tabela 3 se observa o resultado da regressão logística não condicional, pelo método Mantel-Haenszel, para cada um dos modelos.

Tabela 3-Associação entre periodontite e baixo peso ao nascer de acordo com os critérios de definição da periodontite. Salvador, Bahia. (n=299).

\begin{tabular}{lcccc}
\hline Exposições & OR bruta & IC $(95 \%)$ & $\begin{array}{c}\text { OR } \\
\text { ajustada* }\end{array}$ & IC (95\%) \\
\hline $\begin{array}{c}\text { Critério } 1 \\
\text { BPN }\end{array}$ & 0,92 & $(0,561,51)$ & 0,94 & $(0,561,56)$ \\
$\begin{array}{c}\text { Critério 2 } \\
\text { BPN }\end{array}$ & 1,21 & $(0,692,15)$ & 1,18 & $(0,652,13)$ \\
$\begin{array}{c}\text { Critério 3 } \\
\text { BPN }\end{array}$ & 0,87 & $(0,312,43)$ & 1,09 & $(0,373,22)$ \\
\hline
\end{tabular}

Fonte: Autoria própria

\section{DISCUSSÃO}

Embora a literatura aponte evidências da associação entre a periodontite materna e o BPN (CRUZ et al., 2005, GOMES-FILHO et al., 2007, MANNEM; CHAVA, 2011, OFFENBACHER et al., 1996; SADDKI et al., 2008, SANZ; KORNMAN, 2013) neste estudo não foi observada associação estatisticamente significante entre a periodontite e o desfecho gestacional, independentemente do critério utilizado para a definição da doença.

Oito diferentes critérios de definição da periodontite, com ordem crescente de rigor, aplicados a uma amostra de adultos do município de Feira de Santana-Ba, revelaram variações significativas na ocorrência da periodontite, dependentes do critério utilizado. O menor percentual foi observado para o critério mais robusto, de alta especificidade, e que utilizou o maior número de sítios acometidos (GOMES-FILHO et al., 2006), no qual a doença foi definida pela presença de profundidade de sondagem $\geq 4 \mathrm{~mm}$, perda de inserção clínica de $\geq 3 \mathrm{~mm}$ e presença de sangramento no mesmo sítio.

Lopes (2012) avaliou 297 puérperas de maternidades públicas de Salvador-Ba e observou ausência de associação entre a periodontite e o BPN, corroborando os achados deste estudo, mesmo quando avaliada a ação sinérgica com a hiperglicemia não diabética. $O$ critério utilizado para definir a doença periodontal foi bastante rígido e atende às solicitações de Gomes-Filho et al. (2006), por considerar a mensuração no NIC, PS e a presença de sangramento, apresentando alta especificidade e redução de falsos positivos.

A amostra de puérperas avaliada por Lopes (2012) era composta de mulheres jovens, faixa etária na qual a manifestação da doença periodontal é normalmente branda e restrita a poucas unidades e sítios dentários (SUSIN et al., 2011; IDE; PAPAPANOU, 2013). Diante disto, questionou-se, a utilização de um critério de caráter tão restritivo para avaliar esta população e levantou-se a hipótese de que definições da periodontite que considerem mensurações mais flexíveis fossem mais adequadas a esta avaliação.

Apesar dos diferentes critérios utilizados, as medidas de ocorrência da periodontite mostraram-se elevadas em todos os grupos avaliados no presente estudo, variando de $21,29 \%$ (critério 2) a 94,55\% (critério 3). As magnitudes da doença podem ter sido influenciadas pela diferença de robustez e sinais clínicos utilizados nos diferentes critérios, de forma que a maior ocorrência da doença foi observada quando o diagnóstico foi realizado utilizando o critério que empregava um único descritor clínico. 0 
critério mais restritivo, por sua vez, apresentou a menor ocorrência da doença na amostra. No entanto, a periodontite, independente do critério de definição utilizado, não se mostrou associada ao BPN, mesmo após o ajuste pelo número de consultas pré-natais.

Contrariamente, Cruz et al. (2009) mostraram duas vezes mais chances de gerar bebês de baixo peso em mulheres com diagnóstico positivo de periodontite. Associação positiva e estatisticamente significante também foi observada em uma investigação prospectiva de 500 gestantes da Malásia (SADDKI et al., 2008). No referido estudo, e utilizando o critério 2, a ocorrência de baixo peso foi 3,84 vezes superior (IC $95 \%=1.34-11.05$ ) nas gestantes portadoras de periodontite quando realizada a análise multivariada.

O limiar do NIC $\geq 3 \mathrm{~mm}$ foi considerado adequado para a avaliação de populações jovens (MANNEM; CHAVA, 2011; SUSIN et al., 2011), mas também julgado falho (GOMES-FILHO et al., 2007) na identificação de indivíduos verdadeiramente afetados pela periodontite, devido a elevada probabilidade de inclusão de falsos positivos, alta sensibilidade $[100(95,4-100)]$, baixíssima especificidade $[7,9(4,7-12,8)]$ e baixo valor preditivo positivo [35 $(29,5-40,8)]$, quando comparado ao critério 2 .

A flexibilização das definições da periodontite tem sido indicada para a adequação desta ao desenho e à população de um dado estudo. No entanto, descritores que incorram na inclusão de vieses nos resultados e associações analisadas tendem a comprometer, ou mesmo invalidar uma investigação (GOMES-FILHO et al., 2007). Os resultados para o critério 3 , sugerido como uma alternativa às classificações mais rígidas que subestimariam a manifestação caracteristicamente mais branda da doença em populações jovens (SUSIN et al., 2011; IDE; PAPAPANOU, 2013), implicam na possibilidade de ocorrência muito alta da periodontite no momento do exame, além de sugerir a nulidade da associação entre a periodontite e o BPN. Por outro lado, os achados podem revelar uma fragilidade destas mensurações para avaliar a periodontite e, por conseguinte, sua associação com desfechos gestacionais.

O critério 1 (EKE; PAGE, 2012) apresentou a segunda maior ocorrência da periodontite, apontando ausência de saúde periodontal em $41,47 \%$ das mulheres do estudo. Também identificou percentual similar de periodontite crônica entre os grupos caso e controle e não houve significância estatística quando estimada a odds bruta e a ajustada (tabela 3).

Gomes Filho et al. (2007) sugerem a utilização de medidas clínicas combinadas de NIC, PS e SS visando um critério mais restrito, específico e capaz de reduzir a ocorrência de falsos positivos e distorções. No entanto, populações jovens apresentam poucos sítios acometidos por bolsas profundas e por grave perda de inserção (IDE; PAPAPANOU, 2013; SUSIN et al., 2011).

Neste contexto, já era esperado que o critério 2 (GOMES-FILHO et al., 2006), por ser o mais rigoroso, apresentasse a menor ocorrência de periodontite, redu- zindo de forma significativa a proporção de expostos na população. Entretanto, esta foi a definição cujo modelo apresentou a maior medida de associação (tabela 3), ainda que sem significância estatística.

$\mathrm{Na}$ ausência de um consenso quanto a definição ideal da periodontite, assim como dos descritores de medição da sua gravidade e extensão, a significância estatística da associação da doença com resultados gestacionais adversos é diretamente determinada pelos critérios e/ ou medidas clínicas eleitas para cada investigação (GOMES-FILHO et al., 2006; GOMES-FILHO et al., 2007; MANAU et al., 2008). Neste estudo, os critérios 1 e 3 apresentavam limiares mais flexíveis quando comparados ao critério 2 , predispondo, teoricamente, os primeiros a serem mais adequados ao uso em adultos jovens. Todavia, na amostra estudada, a flexibilização destes limiares implicou apenas na variação da ocorrência da doença, mas aparentemente não se garantiu uma avaliação mais adequada à associação em tela, ou seja, o uso de diferentes critérios de definição da exposição não alterou o resultado relativo à associação entre a periodontite e o BPN.

Embora de forma limitada, os achados deste trabaIho suscitam um questionamento acerca da criação e utilização de definições robustas da periodontite para avaliação da sua associação com morbidades sistêmicas, cujo comportamento frente a outras simplificadas e menos específicas se mostrou similar. A sensibilidade e especificidade dos modelos utilizados não foram avaliadas neste trabalho, por não haver um critério que tenha sido considerado comprovadamente superior. Valendo-se também das limitações deste tipo de análise e das incertezas sobre a coerência dos sinais e mensurações atualmente utilizados para avaliar o risco sistêmico decorrente da periodontite, parece razoável propor que investigações futuras concentrem seus esforços em correlacionar os critérios de definição da periodontite com o seu potencial patogênico (perfil microbiológico e imunológico) em lugar de comparar os critérios entre si, visando maior probabilidade de responder as perguntas que cercam estas associações.

Os três critérios diagnósticos avaliados apresentaram definições categóricas e dicotômicas da periodontite, seguindo a indicação de que casos que considerem um desfecho dicotômico são mais adequados ao estudo da associação da periodontite com complicações gestacionais, em detrimento do uso de médias de mensurações clínicas (MANAU et al., 2008). No entanto, Sanz e Kornman (2013) reforçam o uso de medidas clínicas contínuas que permitem avaliar um maior número de condições periodontais potencialmente envolvidas, embora admitam limitações destes descritores no caso de populações onde a extensão da doença é baixa, caso da população analisada neste estudo.

Ide e Papapanou (2013) consideram a combinação destas medidas às categorias da doença a melhor forma de mensuração da periodontite neste contexto, devido ao desconhecimento sobre quais sinais clínicos seriam 
mais adequados para mensurar o dano sistêmico: se os que revelam o histórico acumulado da doença (NIC) ou os que demonstram a carga inflamatória corrente - PS, IG, SS (IDE, PAPAPANOU, 2013; MANAU et al., 2008).

Quatorze diferentes critérios de definição da periodontite e outras 50 mensurações clínicas contínuas foram aplicados a uma população de 1.296 mulheres com idade entre 18 e 40 anos. Após regressão logística não condicional, apenas os casos que utilizaram o NIC entre os descritores clínicos demonstraram significância estatística na associação entre a periodontite e o parto prematuro e o baixo peso ao nascer (MANAU et al., 2008).

O NIC é o descritor de escolha para a mensuração da periodontite na maioria dos estudos epidemiológicos (GOMES-FILHO et al., 2006; GOMES-FILHO et al., 2007; MANAU et al., 2008) embora clinicamente reflita apenas se houve ou não perda de inserção no sítio avaliado, não revelando se, no momento do exame, existe atividade da doença. Apesar dos critérios do presente estudo terem utilizado o NIC para conferir o diagnóstico dos indivíduos (tabela 1), em diferentes limiares e combinações, não se observou significância estatística para a associação entre nenhum deles, rejeitando a hipótese de associação entre a periodontite e o BPN.

O número e a localização dos dentes e sítios avaliados e acometidos, para conferir o diagnóstico, são encontrados em infinitas combinações na literatura, o que também influencia a ocorrência e a magnitude da associação (GOMES-FILHO et al., 2009). Distintos padrões dos descritores foram utilizados no estudo, com avaliação de seis sítios para cada unidade dentária (critério 2) ou apenas dos sítios interproximais (critério 1 e 3 ), cujo uso é controverso. Excluir faces livres poderia desconsiderar sítios verdadeiramente acometidos (BALEUM; LÓPEZ, 2012), contrariamente, a sua inclusão poderia englobar perda de inserção por motivos alheios aos periodontais ${ }^{1}$ Além disso, acredita-se que a doença se inicia e se mostra mais grave nos sitios interproximais (EKE; PAGE, 2012).

Posto que o tempo requerido para o desenvolvimento da periodontite é bastante superior àquele necessário para que se observe clinicamente uma inflamação gengival reversível (LÖE et al., 1965), o período de uma gestação foi julgado insuficiente para causar alteração significante nos exames periodontais realizados no início e ao final da gravidez, sendo considerados equivalentes. Não se constituindo, portanto, uma limitação o diagnóstico da periodontite materna no período pós-parto.

A amostra do estudo originário do presente trabalho foi calculada a partir de dados da literatura (CRUZ et al., 2005) obtendo-se um mínimo de 270 mulheres, com um nível de confiança de $95 \%$ e um poder do estudo de $80 \%$. O desenho do estudo mostrou-se adequado para avaliar a associação entre periodontite materna e o BPN. Todavia, admite-se que a amostra requerida para comparar critérios de diagnóstico da periodontite possa ser superior à utilizada. Considerando indivíduos jovens com baixa extensão e gravidade da periodontite, grande diferença foi observada entre as ocorrências da doença no estudo e a medida utilizada no cálculo do tamanho da amostra, o que pode ter comprometido o poder do estudo (LOPES, 2012).

Além disso, investigações do tipo caso-controle apresentam limitada capacidade de inferir causalidade e determinar novos métodos diagnósticos, entretanto, produzem informações relevantes para o desenvolvimento de pesquisas prospectivas e mais abrangentes.

Diante do desconhecimento dos sinais ideais para definir a periodontite e seu risco sistêmico, os achados deste trabalho demonstram que é imperativa a produção de investigações que estabeleçam uma comparação e trabalhem critérios de definição mais consistentes da periodontite. Sugere-se o uso de medidas clínicas contínuas, definições dicotomizadas e, adicionalmente, dados microbiológicos e imunológicos visando a identificação de sinais clínicos mais adequados para refletir os efeitos sistêmicos da doença e seus potenciais mecanismos de ação. Reduzindo, assim, a existência de vieses e a subestimação da doença e, por conseguinte, o impacto destes problemas na mensuração da ocorrência de eventos sistêmicos em populações jovens.

\section{CONCLUSÃO}

Os diferentes critérios de definição da periodontite analisados neste estudo comportaram-se de forma similar, quando avaliada a associação desta com o BPN em uma população de mulheres jovens. Classificações menos robustas da periodontite não foram capazes de mostrar-se mais adequadas ao uso em indivíduos jovens e as definições mais robustas, com maior número de descritores clínicos, não se sobrepuseram às demais, ocasionando apenas variação na ocorrência da doença. Embora estes achados possam revelar a ausência de associação entre as morbidades estudadas, suscitam questionamento acerca dos critérios criados exclusivamente com base nos sinais que indicam um prognóstico desfavorável para o dente.

\section{REFERÊNCIAS}

CORTELLI, J. R.; CORTELLI, S.C. Periodontite crônica e agressiva: Prevalência subgengival e frequência de Ocorrência de patógenos periodontais. Revista Biociências, [s.I], v. 9, n. 2, p. 91-96, abr./jun. 2003.

CRUZ, S. S. et al. Contribution of periodontal disease in pregnant women as a risk factor for low birth weight. Commun. Dent. Oral Epidemiol., Copenhagen, v. 37, p. 527-533, 2009.

CRUZ, S. S. et al. Doença periodontal materna como fator associado ao baixo peso ao nascer. Rev. Saúde Pública, São Paulo, v. 39, n. 5, p. 782-787, 2005.

EKE, P. I. et al. Update of the case definitions for population-based surveillance of periodontitis. J. Periodontol., Indianapolis, v. 83, n. 12, p. 1449-1454, Dec. 2012.

GOMES-FILHO, I. S. et al. Comparação de critérios que determinam o diagnóstico clínico da doença periodontal. Rev. Odonto Ciênc., Porto Alegre, v. 21, n. 51, p. 77-78, jan./mar. 2006. 
GOMES-FILHO, I. S. et al. Avaliação prospectiva da periodontite materna e baixo peso ao nascer. Revista de Periodontia, São Paulo, v. 19, n. 4, p. $121-128,2009$

GOMES-FILHO, I. S. et al. Exposure measurement in the association between periodontal disease and prematurity/low birth weight. J. Clin. Periodontol., Copenhagen, v. 34, p. 957-963, 2007.

IDE, M.; PAPAPANOU, P. N. Epidemiology of association between maternal periodontal disease and adverse pregnancy outcomes systematic review. J. Periodontol., Indianapolis, v. 84, Supl. 4, p. 181194, 2013.

LÖE, H.; THEILADE, E.; JENSEN, B. Experimental gingivitis in man. Periodontol., Indianapolis, n. 36, p. 177-187, 1965.

LOPES, L. S. Interação biológica entre doença periodontal e hiperglicemia não diabética materna para a ocorrência do baixo peso ao Nascer. 2012. Dissertação (Mestrado)- Universidade Federal da Bahia, Salvador, 2012.

LOURO, P. M. et al. Doença periodontal na gravidez e baixo peso ao nascer. J. Pediatr., Rio de Janeiro, v. 77, n. 1, p. 23-28, 2001.

MANAU, C. et al. Periodontal disease definition may determine the association between periodontitis and pregnancy outcomes. J. Clin. Periodontol., Copenhagen, n. 35, p. 385397, 2008.

MANNEM, S.; CHAVA, V. K. The relationship between maternal periodontitis and preterm low birth weight: A case-control study. Contemp. Clin. Dent., [s.I], v. 2, n. 2, p. 88, Apr./June 2011.

OfFENBACHER, S. et al. Periodontal infection as a possible risk factor for preterm low birth weight. J. Periodontol., Indianapolis, v.67, n. 10, p. 1103-1113, 1996.
ORTELAN, N.; NERI, D. A.; BENICIO, M. H. D. Feeding practices of low birth weight Brazilian infants and associated factors. Rev. Saúde Pública, São Paulo, v. 54, p. 14, 27, jan. 2020.

SADDKI, N. et al. The association between maternal periodontitis and low birth weight infants among Malay women. Community Dent. Oral Epidemiol., Copenhagen, v. 36, p. 296-304, 2008.

SANZ, M.; KORNMAN, K. and on behalf of working group 3 of the joint EFP/AAP workshop. Periodontitis and adverse pregnancy outcomes: consensus report of the Joint EFP/AAP Workshop on Periodontitis and Systemic Diseases. J. Periodontol., Indianapolis, v. 84, Supl. 4, p. 164 $-169,2013$.

SCHENKEIN, H. A. et al. Birth weight of infants of mothers with aggressive periodontitis. J. Periodontol., Indianapolis, v. 83, n. 3, p. 279-296, Mar. 2012.

SUSIN, C. et al. Prevalence and risk indicators for chronic periodontitis in adolescents and young adults in south Brazil. Clin. Periodontol., Copenhagen, v. 38, p. 326-333, 2011.

WORLD HEALTH ORGANIZATION. Infant, Low birth weight. In: Guidelines on optimal feeding of low birth-weight infants in low- and middleincome countries.

Geneva: WHO, 2011. Disponível em: http://www.who.int/maternal_ child_adolescent/documents/9789241548366.pdf. Acesso em: $2 \overline{5}$ Apr.2012.

Submetido em: 05/05/2020

Aceito em: 22/05/2021 